\title{
The Effect of Subject Person in Auxiliary Movement Acquisition by Korean-English Bilingual Children
}

\section{Introduction}

Subject-auxiliary inversion (SAI) in English wh-questions has long been investigated in acquisition studies. Evidence has shown that first language (L1) English learners acquire SAI in stages by auxiliary type (e.g. Rowland \& Pine, 2000, Theakston \& Rowland, 2009) or by whether the wh-element is an argument or adjunct (e.g. de Villiers, 1991, Lee, 2008). The present study provides new evidence that Korean-English (K-E) sequential bilingual children show an effect of person, [PRS], in their acquisition of SAI. The analysis also gives a theoretical account within the Minimalist Program for the observed patterns in the data.

Park (2008) showed that in naturalistic data, K-E bilingual children exhibited a pattern of SAI that was not predictable by auxiliary type or the argument/adjunct distinction. Instead, the data suggested an effect of [PRS] on SAI, such that wh-questions with second person subjects had a higher frequency of SAI use than those with third person subjects. The present study addresses this question of the effect of [PRS], which is stated in (1). Secondly, if there is indeed an effect of person, it is important to account for this pattern of data within the current theory, in which two seemingly unrelated grammatical elements such as auxiliary movement and [PRS] are involved, within the current theory, a question stated in (2).

(1) Is person [PRS] a significant factor in predicting the presence or absence of SAI in English wh-questions for K-E children?

(2) How can this pattern of acquisition be accounted for in generative syntactic theory?

\section{Methodology}

To test the possible effect of [PRS], $21 \mathrm{~K}-\mathrm{E}$ bilingual and 19 monolingual Englishspeaking (M-E) preschool-aged children were recruited for an elicitation study testing the effect of [PRS] on the use of SAI in English wh-questions. The K-E group ranged in age from 4;0 to 6;10 (mean 5;4), and the M-E group ranged from 3;0 to 5;1 (mean 4;2). The K-E group consists of sequential bilingual children, in which they acquire Korean from birth and are exposed to English upon entering preschool (mean age 2;6). The groups were matched on English proficiency through the sentence imitation subtest of the Test of Language Development: Primary - Third Edition (TOLD:P-3).

The experiment tested the factors of subject person (first, second, third) and auxiliary type (AUX-do and AUX-be) in a 3 x 2 design. All target utterances were singular, object-what, wh-questions. Sixty questions were elicited over two separate sessions through a game with puppets, with one examiner to prompt the child and another to operate the puppets. At the start of each sessions was a warm-up period in which children had the opportunity to interact with Teddy, the main puppet, and is introduced to Giraffe, a shy puppet who is too timid to talk to children and can only talk to Teddy. After the warm-up, the children were given three practice items. If the children could not complete these practice items even with scripted hints provided, the task would be stopped.

First person questions were elicited by placing three objects in front of the child. The Storyteller would demonstrate an action, e.g. brush, then prompt the child to choose one of the three objects on which to repeat the action while Teddy's eyes were closed. Once the child was finished, the child was prompted Ask Teddy what you brushed, which elicits the target question 
What did I brush. For AUX-be, the child was prompted Ask Teddy what you were brushing. Second person questions were elicited by Teddy doing the action where the child could not see the direct object of the verb. The child was prompted, Ask Teddy what he brushed, eliciting the target What did you brush? For AUX-be, the child was prompted Ask Teddy what he was brushing. For third person questions, shy Giraffe would brush something while Teddy watched. Because Giraffe is shy, the child would have to ask Teddy about her. The child was prompted, Ask Teddy what she brushed, eliciting What did she brush. For AUX-be, the child was prompted Ask Teddy what she was brushing.

This procedure was repeated using ten different monosyllabic, high-frequency verbs from ChildFreq (Kungshuset, 2010). Six different forms of the test with counterbalanced items was used to reduce possible ordering effects. For each of the 60 items, the child's response on auxiliary movement was recorded as YES if the auxiliary occurred above the subject, and NO if the auxiliary was either below the subject or not present. A generalized linear mixed model was used for the quantitative analysis of children's responses on SAI.

\section{Results}

Results indicated a main effect for group, $F(1,2116)=16.33, p<.0001$. Due to the expected difference between the two groups on SAI use, the groups were analyzed separately for the effect of person on auxiliary movement. The K-E group showed a significant effect for subject person on SAI, $F(2,1087)=34.25, p<.0001$, while no effect of person was found for the M-E group, $F(2,1026)=2.50, p=.08$. This finding indicates that the value of [PRS] has a significant effect on the appearance or absence of SAI for the bilingual group, while no such effect can be found for the monolingual English group. Posthoc analysis of K-E responses also revealed significant differences between each of the person values, such that SAI occurred significantly more for second person than for first person, $t(1087)=5.56, p<.0001$, and for third person, $t(1096)=-8.18, p<.0001$.

Furthermore, qualitative analysis of K-E productions revealed four stages of SAI acquisition that is predictable based on the children's score on the TOLD:P-3 test. These stages are as follows: 1) no auxiliary present, 2) SAI for second person, 3) SAI for first person, and 4) SAI for third person. This was evident for both do and be. Examples from each stage can be seen in Tables 1 for AUX-do.

Table 3. Stages of AUX-do Acquisition

\begin{tabular}{lllcl} 
Stage & Participants $(n)$ & Mean Age & Mean TOLD:P-3 & Examples \\
\hline 1 & 6 & $4 ; 5$ & 1.0 & $\begin{array}{l}\text { What I clean? (109-C) } \\
\text { What you cook? } \\
\text { What she draw? }\end{array}$ \\
\hline 2 & 2 & & $\begin{array}{l}\text { What I push? (113-A) } \\
\text { What'd you cook? } \\
\text { What she wash? }\end{array}$ \\
\hline 3 & 2 & 1.5 & $\begin{array}{l}\text { What did I drink? (110-D) } \\
\text { What did you hold? } \\
\text { What she brush? }\end{array}$ \\
\hline 4 & 11 & $6 ; 0$ & 3.5 & $\begin{array}{l}\text { What did I clean? (106-F) } \\
\text { What did you wash? } \\
\text { What did she drink? }\end{array}$ \\
\hline
\end{tabular}


This step-wise development was also seen for AUX-be, with an added stage in which children produced be in situ before entering Stage 2, such as What you were eating? These patterns in which children develop SAI based on [PRS] is consistent with what the quantitative results show, in that there is a significant difference between each person value. The contrasts at every level are very distinct, as all ten items within first, second, and third person appear consistently with or without SAI, with little to no deviation from this pattern.

\section{Analysis}

To theoretically account for the patterns seen in the results from the experiment in which [PRS] significantly affects the presence of SAI for K-E children, the Person Feature Auxiliary Movement (PFAM) Hypothesis is proposed, as in (3).

(3) The Person-Feature Auxiliary Movement Hypothesis
a.

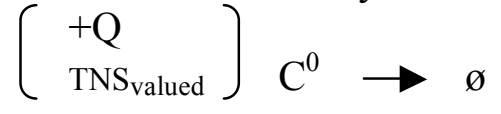
b. $\left(\begin{array}{l}+\mathrm{Q} \\ \mathrm{TNS}_{\text {valued }} \\ \mathrm{PRS}\end{array} \mathrm{C}^{0} \rightarrow\right.$ do

The PFAM Hypothesis, combining feature composition with basic elements of Distributed Morphology, proposes that the combination of features in (3a) results in a null form in Spell Out, while the features in (3b) will result in $d o$ for Korean-English bilingual children. In other words, the presence or lack of the [PRS] feature is responsible for the appearance of variable behavior of T-to-C movement in wh-questions.

(4) PFAM Hypothesis Part A

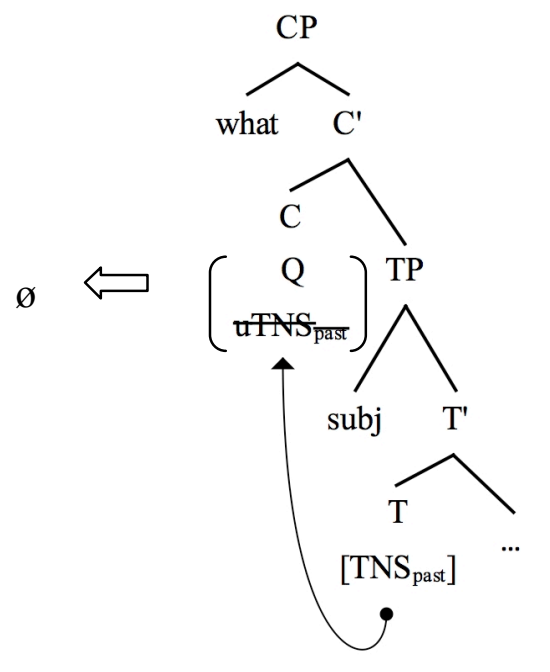

(5) PFAM Hypothesis Part B

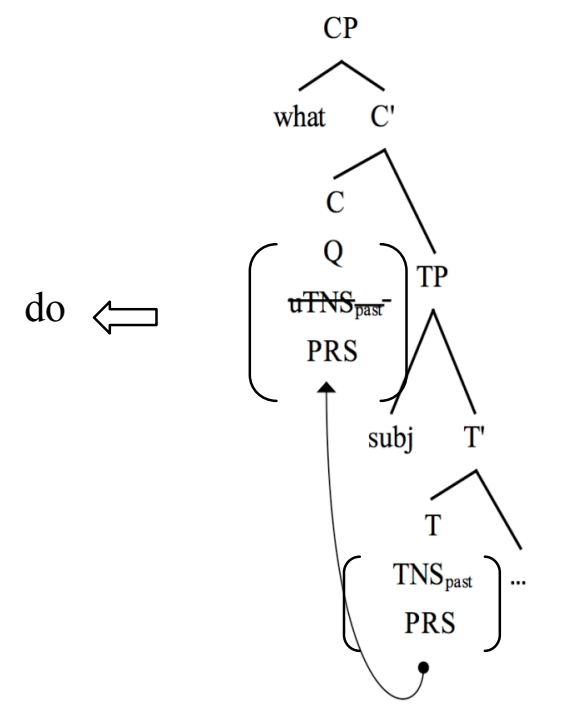

Before [PRS] is acquired, children lack the feature in $\mathrm{T}$ altogether, such that the head of $\mathrm{T}$ consists of only the [TNS] feature, which is allowed to check the [uTNS] feature in C singly. This is Spelled Out in the head of $\mathrm{C}$ as a phonologically null element, as seen in (4). However, as 
each feature value is acquired for second, first, and third person, the feature [PRS] becomes present in T. Due to the presence of this specified feature in the head of T, the [TNS] feature may not be raised to $\mathrm{C}$ singly without breaking morphological integrity of the complex head of $\mathrm{T}$, thereby necessitating pied-piping of the entire matrix of features that form it. This is Spelled Out as $d o$, as in (5). Based on the qualitative data that showed a stepwise development of SAI based on [PRS], I propose that initially, Korean-English bilingual children have not yet acquired the person feature [PRS]. Note that it is not T-to-C movement that children are lacking, but rather that there is simply no condition for the auxiliary to appear in C. As each value of [PRS] is acquired and becomes present in $\mathrm{T}$, the auxiliary became visible in $\mathrm{C}$ as a result of the piedpiping of the T-matrix, containing the [PRS] feature.

To further elaborate on the [PRS] feature, we must consider that in English, a three-way distinction for person must be established. This has been accomplished by expanding [PRS] into two features, [ \pm ADDRESSEE] and [ \pm PARTICIPANT] (Nevin, 2007). Under such a system, I propose that K-E children acquire [ADDRESSEE] as a privative feature first, which leads to postulate only second person at this time. The nature of privative features is such that when they are not present, they are simply not part of the derivation. Thus, operations may not target them, i.e. they will not be triggers for certain operations. Crucially, a child may acquire [ADDRESSEE] initially, but all others that do not have this feature are treated as non-existent, until the whole binary value system (with plus and minus value) is acquired. In the next stage, children postulate a binary feature system for that feature [ \pm ADDRESSEE], which yields the first and second person distinction, but still no specification for third person. Finally, children acquire a binary distinction for [ \pm PARTICIPANT], resulting in specification for third person. This system of acquiring each feature subsequently gives rise to a second $>$ first $>$ third sequence of acquisition, as observed in the data.

To conclude, this paper presents new evidence for a link between person and SAI for Korean-English bilingual children that is not seen in monolingual English-speaking children. Additionally, the paper posits a theoretical account of the results, through the Person Feature Auxiliary Movement Hypothesis, which links [PRS] and T-to-C movement, two seemingly unrelated elements. The PFAM sheds light on the impact of features in the head of $\mathrm{T}$ and the appearance of movement to the head of CP. Also, the analysis shows that K-E children have access to T-to-C movement from the earliest stages, crediting bilingual children with full competence of syntactic operations.

\section{References}

de Villiers, J. (1991). Why questions? In T. Maxfield \& B. Plunkett (Eds.), Papers in the acquisition of WH (Vol. University of Massachusetts Occasional Papers, pp. 155-173). Amherst.

Lee, S.-Y. (2008). Argument-adjunct asymmetry in the acquisition of inversion in wh-questions by Korean learners of English. Language Learning, 58(3), 625-663. doi: 10.1111/j.1467-9922.2008.00452.x

Kungshuset, L. (2010). ChildFreq. Retrieved 8 Jun 2010

Nevin, A. (2007). The representation of third person and its consequences for person-case effects. Natural Language \& Linguistic Theory, 25, 273-313.

Park, S. K. (2008). The Acquisition of Wh-questions by Korean-English Bilingual Children: The Role of Crosslinguistic Influence. Master of Arts Master's Thesis, Purdue University, West Lafayette, IN.

Rowland, C., \& Pine, J. (2000). Subject-auxiliary inversion errors and wh-question acquisition: 'what children do know?' Journal of Child Language, 27(1), 157-181.

Theakston, A., \& Rowland, C. (2009). The acquisition of auxiliary syntax: A longitudinal elicitation study. Part 1: Auxiliary BE. Journal of Speech, Language, and Hearing Research, 52(6), 1449-1470. doi: 10.1044/10924388(2009/08-0037) 